\title{
Existence and asymptotic of traveling wave fronts for the delayed Volterra-type cooperative system with spatial diffusion
}

Yanling Meng ${ }^{1}$, Weiguo Zhang ${ }^{2 *}$ and Zhixian $\mathrm{Yu}^{2}$

"Correspondence: zwgzss@163.com; zwgzwm@126.com

${ }^{2}$ College of Science, University of Shanghai for Science and Technology, Shanghai, China Full list of author information is available at the end of the article

\section{算 Springer}

\begin{abstract}
In the paper, we are concerned with the existence and the exponential asymptotic behavior of traveling waves for the delayed Volterra-type cooperative system with nonquasimonotone condition

$$
\left\{\begin{array}{l}
\frac{\partial u_{1}(x, t)}{\partial t}=D_{1} \frac{\partial^{2} u_{1}(x, t)}{\partial x^{2}}+r_{1} u_{1}(x, t)\left[1-a_{1} u_{1}(x, t)-b_{1} u_{1}\left(x, t-\tau_{1}\right)+c_{1} u_{2}\left(x, t-\tau_{2}\right)\right], \\
\frac{\partial u_{2}(x, t)}{\partial t}=D_{2} \frac{\partial^{2} u_{2}(x, t)}{\partial x^{2}}+r_{2} u_{2}(x, t)\left[1-a_{2} u_{2}(x, t)-b_{2} u_{2}\left(x, t-\tau_{3}\right)+c_{2} u_{1}\left(x, t-\tau_{4}\right)\right],
\end{array}\right.
$$
\end{abstract}

modeling the variation of the populations. Here, the major contribution to population model is the introduction of population self-regulation depending on not only the populations at time $t$, but also on the earlier population time $t-\tau_{i}(i=1,3)$. By constructing a pair of suitable upper and lower solutions we obtain the existence of traveling wave fronts connecting the trivial equilibrium and the positive equilibrium, which indicates that there is a transition zone moving the steady state with no species to the steady state with the coexistence of two species. Furthermore, with the help of Ikehara's theorem, the exponential asymptotic behavior of traveling wave front is exactly derived for this system without quasi-monotone conditions. The results are not only an extension of existing results for the known logistic or cooperative system, but also can extend another type of delayed logistic equation with spatial diffusion

$$
\frac{\partial u(x, t)}{\partial t}=D \frac{\partial^{2} u(x, t)}{\partial x^{2}}+r u(x, t)[1-a u(x, t)-b u(x, t-\tau)] .
$$

MSC: 35C07; 92D25; 35B35

Keywords: Nonquasimonotone; Volterra-type; Cooperative system; Traveling wave front; Asymptotic behavior

\section{Introduction}

The first model of interacting populations to be considered is a classical one, usually associated with the names of Lotka and Volterra. One of the simplest models of the dynamics of two interacting mutualistic species was in the form of an autonomous system

(c) The Author(s) 2018. This article is distributed under the terms of the Creative Commons Attribution 4.0 International License (http://creativecommons.org/licenses/by/4.0/), which permits unrestricted use, distribution, and reproduction in any medium, provided you give appropriate credit to the original author(s) and the source, provide a link to the Creative Commons license, and indicate if changes were made. 
of ordinary differential equations with quadratic nonlinearities,

$$
\left\{\begin{array}{l}
\frac{d u_{1}(t)}{d t}=r_{1} u_{1}(t)\left[1-b_{1} u_{1}(t)+c_{1} u_{2}(t)\right], \\
\frac{d u_{2}(t)}{d t}=r_{2} u_{2}(t)\left[1-b_{2} u_{2}(t)+c_{2} u_{1}(t)\right],
\end{array}\right.
$$

where $b_{i}, c_{i}, r_{i}(i=1,2)$ are positive constants, $u_{i}, i=1,2$, are the numbers of individuals of species $i, K_{i}=\frac{1}{b_{i}}, i=1,2$, are the carrying capacities of species $i, r_{i}, i=1,2$, are the intrinsic rates of increase of species $i, c_{1}\left(c_{2}\right)$ measures the rate at which an individual of $u_{2}\left(u_{1}\right)$ benefits the growth rate of population $u_{1}\left(u_{2}\right)$. We refer to $[1,20,26,30,42]$ for the details.

Time delays in the growth dynamics of a population or of several interacting species can arise from a great variety of causes and are undoubtedly always present to some extent; see $[3,18,37,42]$. Certainly, the most famous example is the familiar Volterra-type logistic equation with infinite time delay

$$
\frac{d u(t)}{d t}=u(t)\left[b-a u(t)-d \int_{-\infty}^{t} u(s) k(t-s) d s\right]
$$

which means that the growth rates at time $t$ are effected by population sizes at (possibly all) previous times $s \leq t$ distributed (or weighted) in the past by the delay kernel $k(s)$, where $a$, $b, d$ are positive constants. Cushing [3] investigated the asymptotic behavior (as $t \rightarrow+\infty$ ) of solutions or, more specifically, of topics such as the stability or instability of equilibria and the oscillatory nature of solutions. Seifert [29] obtained asymptotic behavior results by using different methods for a particular case of (1.2)

$$
\frac{d u(t)}{d t}=u(t)[b-a u(t)-d u(t-1)]
$$

which arises in models for the variation of the population of species where the death rate depends on not only the population at time $t$, but also on the population one unit of earlier time. Gopalsamy [7] also studied the asymptotic behavior (as $t \rightarrow+\infty$ ) of solutions for a logistic equation with two delays

$$
\frac{d u(t)}{d t}=u(t)\left[b-a u\left(t-\tau_{1}\right)-d u\left(t-\tau_{2}\right)\right]
$$

where $\tau_{1}, \tau_{2}$ are positive constants.

To specify an interacting species model, the spatial dispersal interactions often cannot be neglected (see $[1,26])$. The theory of traveling wave solutions for the system with spatial dispersal is one of the fast developing areas of modern mathematics. Traveling wave solutions are solutions of special type. They can be usually characterized as the solutions invariant with respect to the translation in space. The most frequently used model for studying the spread of a single population in a homogenous environment is the Fisher equation

$$
\frac{\partial u(x, t)}{\partial t}=D \frac{\partial^{2} u(x, t)}{\partial x^{2}}+r u(x, t)[1-a u(x, t)]
$$

introduced by Fisher [5], where $a, r, D$ are positive constants. Fisher found that (1.5) has a traveling wave solution $u(x, t)=\phi(x+c t)$ with speed $c \geq 2 \sqrt{D r}$. We refer to $[17,26,34]$ 
for more detail. In the case where there are more than one species, we can incorporate the interaction between the species to obtain the so-called Lotka-Volterra-type diffusive model. The interaction can be either competitive or cooperative. Some related problems on traveling wave solutions for the Lotka-Volterra competitive system with spatial diffusion

$$
\left\{\begin{array}{l}
\frac{\partial u_{1}(x, t)}{\partial t}=D_{1} \frac{\partial^{2} u_{1}(x, t)}{\partial x^{2}}+r_{1} u_{1}(x, t)\left[1-a_{1} u_{1}(x, t)-b_{1} u_{2}(x, t)\right] \\
\frac{\partial u_{2}(x, t)}{\partial t}=D_{2} \frac{\partial^{2} u_{2}(x, t)}{\partial x^{2}}+r_{2} u_{2}(x, t)\left[1-a_{2} u_{2}(x, t)-b_{2} u_{1}(x, t)\right]
\end{array}\right.
$$

were investigated in $[8-10,14-16,31,33]$ and the references therein, where $a_{i}, b_{i}, r_{i}, D_{i}$ $(i=1,2)$ are positive constants. For the Lotka-Volterra competitive system with delays and spatial diffusion, the existence of traveling wave solutions was also admitted in [4, $21,22,25,40]$. Huang and Zou [12] naturally considered the system with two mutualistic species

$$
\left\{\begin{array}{l}
\frac{\partial u_{1}(x, t)}{\partial t}=D_{1} \frac{\partial^{2} u_{1}(x, t)}{\partial x^{2}}+r_{1} u_{1}(x, t)\left[1-b_{1} u_{1}\left(x, t-\tau_{1}\right)+c_{1} u_{2}\left(x, t-\tau_{2}\right)\right] \\
\frac{\partial u_{2}(x, t)}{\partial t}=D_{2} \frac{\partial^{2} u_{2}(x, t)}{\partial x^{2}}+r_{2} u_{2}(x, t)\left[1-b_{2} u_{2}\left(x, t-\tau_{3}\right)+c_{2} u_{1}\left(x, t-\tau_{4}\right)\right]
\end{array}\right.
$$

where $a_{i}, b_{i}, r_{i}, D_{i}(i=1,2)$ and $\tau_{j}(j=1,2,3,4)$ are positive constants. By using the technique developed by Wu and Zou [38], they obtained that, for every $c>2 \max \left\{\sqrt{D_{1} r_{1}}\right.$, $\left.\sqrt{D_{2} r_{2}}\right\},(1.7)$ has a monotone traveling wave solution (i.e., a traveling wave front), which connects $(0,0)$ to

$$
\left(\frac{b_{2}+c_{1}}{b_{1} b_{2}-c_{1} c_{2}}, \frac{b_{1}+c_{2}}{b_{1} b_{2}-c_{1} c_{2}}\right)
$$

provided that $\tau_{1}$ and $\tau_{3}$ are sufficiently small and $b_{1} b_{2}>c_{1} c_{2}$.

Motivated by the works mentioned, we consider the revised delayed Volterra-type cooperative system with spatial diffusion

$$
\left\{\begin{array}{l}
\frac{\partial u_{1}(x, t)}{\partial t}=D_{1} \frac{\partial^{2} u_{1}(x, t)}{\partial x^{2}}+r_{1} u_{1}(x, t)\left[1-a_{1} u_{1}(x, t)-b_{1} u_{1}\left(x, t-\tau_{1}\right)+c_{1} u_{2}\left(x, t-\tau_{2}\right)\right], \\
\frac{\partial u_{2}(x, t)}{\partial t}=D_{2} \frac{\partial^{2} u_{2}(x, t)}{\partial x^{2}}+r_{2} u_{2}(x, t)\left[1-a_{2} u_{2}(x, t)-b_{2} u_{2}\left(x, t-\tau_{3}\right)+c_{2} u_{1}\left(x, t-\tau_{4}\right)\right]
\end{array}\right.
$$

where $r_{i}>0, a_{i}>0, b_{i}>0, c_{i}>0, i=1,2, \tau_{j}>0, j=1,2,3,4$. Here, the major contribution to population modeling is the introduction of population self-regulation depending not only on the population at time $t$, but also on the population at earlier time $t-\tau_{i}(i=1,3)$. Thus, it is a very interesting and important mathematical problem to investigate its dynamics on traveling wave solutions. In this paper, we mainly study the existence and exponential asymptotic behavior of traveling wave fronts for (1.8).

To obtain the existence of traveling wave fronts connecting the trivial equilibrium $(0,0)$ to the positive equilibrium

$$
\left(\frac{a_{2}+b_{2}+c_{1}}{\left(a_{1}+b_{1}\right)\left(a_{2}+b_{2}\right)-c_{1} c_{2}}, \frac{a_{1}+b_{1}+c_{2}}{\left(a_{1}+b_{1}\right)\left(a_{2}+b_{2}\right)-c_{1} c_{2}}\right),
$$

we still adopt the theory in $[13,38]$ by constructing a pair of suitable upper and lower solutions. We notice that the result is an extension of the existing results for the known logistic 
or cooperative system. For example, letting $a_{1}=a_{2}=0$, (1.8) reduces to system (1.7) investigated by Huang and Zou [12]. Especially, taking $c_{1}=c_{2}=0,(1.8)$ can be decoupled into another type of two delayed logistic scaler equations with spatial diffusion

$$
\frac{\partial u(x, t)}{\partial t}=D \frac{\partial^{2} u(x, t)}{\partial x^{2}}+r u(x, t)[1-a u(x, t)-b u(x, t-\tau)]
$$

where $a \geq 0, b \geq 0, D \geq 0, r \geq 0, \tau>0$. It is obvious that (1.9) reduces to (1.5) by choosing the parameter $b=0$ or $\tau=0$. Taking the parameter $a=0$, (1.9) reduces to the delayed logistic equation

$$
\frac{\partial u(x, t)}{\partial t}=D \frac{\partial^{2} u(x, t)}{\partial x^{2}}+r u(x, t)[1-b u(x, t-\tau)]
$$

which was widely studied in $[6,19,28,38]$.

To our knowledge, the exponential asymptotic behavior at negative infinity of traveling wave fronts for (1.7) cannot be obtained by construction of the upper and lower solutions; see [12]. Notice that the exponential asymptotic behavior of traveling wave fronts for kinds of equations with quasimonotone conditions is widely investigated (see $[2,11,35,39$, 41]). For a nonquasimonotone equation, Pan [28] obtained the exponential asymptotic behavior at negative infinity of traveling wave fronts. However, for nonquasimonotone systems, the exponential asymptotic behavior of traveling wave fronts is still open. In this paper, we also answer this problem for system (1.9) with the help of Ikehara's theorem.

The rest of our paper is organized as follow. Section 2 is devoted to recalling a known abstract result. In Sect. 3, we establish the existence of traveling wave fronts for (1.8) by applying a known result. In Sect. 4, we investigate the exponential asymptotic behavior at negative infinity of any traveling wave fronts for (1.7)-(1.9).

\section{An abstract theory}

In the remainder of this paper, we use the usual notation for the standard ordering in $\mathbb{R}^{n}$, that is, for $u=\left(u_{1}, \ldots, u_{n}\right)^{T}$ and $v=\left(v_{1}, \ldots, v_{n}\right)^{T}$, we denote $u \leq v$ if $u_{i} \leq v_{i}, i=1,2, \ldots, n$, and $u<v$ if $u \leq v$ but $u \neq v$. In particular, if $u \leq v$, then we denote $(u, v]=\left\{w \in \mathbb{R}^{n}: u<\right.$ $w \leq v\},[u, v)=\left\{w \in \mathbb{R}^{n}: u \leq w<v\right\}$, and $[u, v]=\left\{w \in \mathbb{R}^{n}: u \leq w \leq v\right\}$. Let $|\cdot|$ denote the Euclidean norm in $\mathbb{R}^{n}$ and $\|\cdot\|$ denote the supremum norm in $C\left([-\tau, 0], \mathbb{R}^{n}\right)$.

We first consider the following reaction-diffusion system with time delay:

$$
\frac{\partial u(x, t)}{\partial t}=D \frac{\partial^{2} u(x, t)}{\partial x^{2}}+f\left(u_{t}(x)\right)
$$

where $t \geq 0, x \in \mathbb{R}, u \in \mathbb{R}^{n}, D=\operatorname{diag}\left(D_{1}, D_{2}, \ldots, D_{n}\right)$ with $D_{i}>0(i=1, \ldots, n), f$ : $C\left([-\tau, 0] ; \mathbb{R}^{n}\right) \rightarrow \mathbb{R}^{n}$ is continuous, and $u_{t}(x)$ is parameterized in $C\left([-\tau, 0] ; \mathbb{R}^{n}\right)$ by $x \in \mathbb{R}$ and given by

$$
u_{t}(x)(\theta)=u(x, t+\theta), \quad \theta \in[-\tau, 0] .
$$

A traveling wave solution of (2.1) is a special translation invariant solution of the form $u(x, t)=\phi(x+c t)$. Here $\phi \in C^{2}\left(\mathbb{R}, \mathbb{R}^{n}\right)$ is the wave profile that propagates through the onedimensional spatial domain at constant velocity $c>0$. Substituting $\phi(x+c t)$ into $(2.1)$ and 
letting $\xi=x+c t$, we have the corresponding wave profile equation

$$
D \phi^{\prime \prime}(\xi)-c \phi^{\prime}(\xi)+f_{c}\left(\phi_{\xi}\right)=\mathbf{0} \quad \text { for } \xi \in \mathbb{R}
$$

where $f_{c}: X_{c}=C\left([-c \tau, 0] ; \mathbb{R}^{n}\right) \rightarrow \mathbb{R}^{n}$ is defined by

$$
f_{c}(\phi)=f\left(\phi^{c}\right), \quad \phi^{c}(s)=\phi(c s), \quad s \in[-\tau, 0] .
$$

If (2.2) has a monotone solution $\phi$ defined on $\mathbb{R}$ for $c>0$ and the limits

$$
\lim _{\xi \rightarrow-\infty} \phi(\xi)=\mathbf{0}, \quad \lim _{\xi \rightarrow+\infty} \phi(\xi)=\mathbf{K}
$$

exist, where $0:=(0, \ldots, 0)$ and $K:=\left(k_{1}, \ldots, k_{n}\right)$ are two equilibria, then $u(x, t)=\phi(x+c t)$ is called a traveling wave front of (2.1) with wave speed $c$. Therefore, (1.8) has a traveling wave front if and only if (2.2) has a solution on $\mathbb{R}$ satisfying the asymptotic boundary condition (2.3).

To tackle the existence of traveling wave fronts of (1.8), suppose that the reaction term $f$ satisfies the following exponential quasimonotonicity condition:

$\left(\mathrm{QM}^{*}\right)$ There exists a matrix $\beta=\operatorname{diag}\left(\beta_{1}, \ldots, \beta_{n}\right)$ with $\beta_{i} \geq 0, i=1, \ldots, n$, such that

$$
f(\phi)-f(\psi)+\beta[\phi(0)-\psi(0)] \geq \mathbf{0}
$$

for $\phi:=\left(\phi_{1}, \ldots, \phi_{n}\right), \psi:=\left(\psi_{1}, \ldots, \psi_{n}\right) \in X=C\left([-\tau, 0]\right.$; $\left.\mathbb{R}^{n}\right)$ with (i) $\mathbf{0} \leq \psi(s) \leq \phi(s) \leq \mathbf{K}$ for $s \in[-\tau, 0]$, (ii) $e^{\beta s}[\phi(s)-\psi(s)]$ is nondecreasing in $s \in[-\tau, 0]$, that is, $e^{\beta_{i} s}\left(\phi_{i}(s)-\psi_{i}(s)\right)$ is nondecreasing in $s \in[-\tau, 0], i=1,2, \ldots, n$.

To state a known result, we introduce the following hypotheses:

(H1) $f(\hat{\mathbf{0}})=f(\hat{\mathbf{K}})=\mathbf{0}$ with $\mathbf{0}<\mathbf{K}$, where, for any $u \in \mathbb{R}^{n}, \hat{u}$ denotes the constant value function on $[-\tau, 0]$ taking the value $u$.

(H2) There is a constant $L>0$ such that

$$
|f(\phi)-f(\psi)| \leq L\|\phi-\psi\|
$$

for $\phi, \psi \in C\left([-\tau, 0], \mathbb{R}^{n}\right)$ with $\mathbf{0} \leq \phi(s), \psi(s) \leq \mathbf{K}, s \in[-\tau, 0]$.

Now we define upper and lower solutions for (2.2).

Definition 2.1 A continuous function $\phi: \mathbb{R} \rightarrow \mathbb{R}^{n}$ is called an upper solution of (2.2) if $\phi^{\prime}$ and $\phi^{\prime \prime}$ exist almost everywhere (a.e.) in $\mathbb{R}$, they are essentially bounded on $\mathbb{R}$, and the following inequality holds:

$$
D \phi^{\prime \prime}(\xi)-c \phi^{\prime}(\xi)+f_{c}\left(\phi_{\xi}\right) \leq \mathbf{0} \quad \text { a.e. in } \mathbb{R}
$$

A lower solution of (2.2) is defined in a similar way by reversing the inequality in (2.4).

In what follows, we assume that (2.2) has an upper solution $\bar{\Phi}$ and a lower solution $\underline{\Phi}$ satisfying the following hypotheses:

(P1) $\mathbf{0} \leq \underline{\Phi}(\xi) \leq \bar{\Phi}(\xi) \leq \mathbf{K}$ and $\sup _{t \leq \xi} \Phi(t) \leq \bar{\Phi}(\xi)$ for $\xi \in \mathbb{R}$; 
(P2) $f(\hat{u}) \neq \mathbf{0}$ for $\hat{u} \in\left(\mathbf{0}, \inf _{\xi \in \mathbb{R}} \bar{\Phi}(\xi)\right] \cup\left[\sup _{\xi \in \mathbb{R}} \Phi(\xi), \mathbf{K}\right)$;

(P3) $e^{\beta \xi}[\bar{\Phi}(\xi)-\underline{\Phi}(\xi)]$ are nondecreasing in $\xi \in \mathbb{R}$.

Define

$$
C_{[\mathbf{0}, \mathbf{K}]}\left(\mathbb{R}, \mathbb{R}^{n}\right)=\left\{\phi: \phi \in C\left(\mathbb{R}, \mathbb{R}^{n}\right) \mid \mathbf{0} \leq \phi \leq \mathbf{K}\right\}
$$

and the profile set

$$
\Gamma^{*}(\underline{\Phi}, \bar{\Phi})=\left\{\begin{array}{ll}
\text { (i) } \quad & \Phi(\xi) \leq \Phi(\xi) \leq \bar{\Phi}(\xi) \text { and is nondecreasing } \\
& \text { in } \xi \in \mathbb{R} . \\
& \text { (ii) } \quad e^{\beta \xi}[\Phi(\xi)-\Phi(\xi)], e^{\beta \xi}[\Phi(\xi)-\underline{\Phi}(\xi)] \\
& \text { are nondecreasing in } \xi \in \mathbb{R} . \\
\text { (iii) } \quad & e^{\beta \xi}[\Phi(\xi+s)-\Phi(\xi)] \text { is nondecreasing in } \\
& \xi \in \mathbb{R} \text { for every } s>0 .
\end{array}\right\}
$$

Now we recall the following result in [13].

Theorem 2.1 Assume that (H1)-(H2) and $\left(\mathrm{QM}^{*}\right)$ hold. If (2.2) has an upper solution $\bar{\Phi}(\xi)$ in $\Gamma^{*}(\underline{\Phi}, \bar{\Phi})$ and a lower solution $\Phi(\xi)$ (which is not necessarily in $\Gamma^{*}(\underline{\Phi}, \bar{\Phi})$ ) satisfying hypotheses (P1)-(P3), then (2.2)-(2.3) have a solution $\Phi \in \Gamma^{*}(\Phi, \bar{\Phi})$, that is, (2.1) has a traveling wave front $\Phi(\xi)$ satisfying $\Phi(-\infty)=\mathbf{0}$ and $\Phi(+\infty)=\mathbf{K}$.

Remark 2.1 According to the proof in [23, 27], we can obtain that the traveling wave front $\Phi(\xi)$ satisfies $\Phi(\xi) \leq \Phi(\xi) \leq \bar{\Phi}(\xi)$ for all $\xi \in \mathbb{R}$.

\section{Existence of traveling wave fronts}

Assume that $\left(a_{1}+b_{1}\right)\left(a_{2}+b_{2}\right)-c_{1} c_{2}>0,(1.8)$ has four equilibria: $(0,0),\left(0, \frac{1}{a_{2}+b_{2}}\right),\left(\frac{1}{a_{1}+b_{1}}, 0\right)$, and

$$
\left(k_{1}, k_{2}\right):=\left(\frac{a_{2}+b_{2}+c_{1}}{\left(a_{1}+b_{1}\right)\left(a_{2}+b_{2}\right)-c_{1} c_{2}}, \frac{a_{1}+b_{1}+c_{2}}{\left(a_{1}+b_{1}\right)\left(a_{2}+b_{2}\right)-c_{1} c_{2}}\right) .
$$

We are mainly interested in the existence of traveling wave fronts connecting the trivial equilibrium $0:=(0,0)$ to the positive equilibrium $\mathbf{K}:=\left(k_{1}, k_{2}\right)$, which indicates that there is a transition zone moving the steady state with no species to the steady state with the coexistence of two species. Substituting $u_{1}(x, t)=\phi_{1}(x+c t)$ and $u_{2}(x, t)=\phi_{2}(x+c t)$ into (1.8) and denoting the moving variable $x+c t$ by $\xi$, we can reduce (1.8) to the following wave profile equations:

$$
\left\{\begin{array}{l}
D_{1} \phi_{1}^{\prime \prime}(\xi)-c \phi_{1}^{\prime}(\xi)+r_{1} \phi_{1}(\xi)\left[1-a_{1} \phi_{1}(\xi)-b_{1} \phi_{1}\left(\xi-c \tau_{1}\right)+c_{1} \phi_{2}\left(\xi-c \tau_{2}\right)\right]=0 \\
D_{2} \phi_{2}^{\prime \prime}(\xi)-c \phi_{2}^{\prime}(\xi)+r_{2} \phi_{2}(\xi)\left[1-a_{2} \phi_{2}(\xi)-b_{2} \phi_{2}\left(\xi-c \tau_{3}\right)+c_{2} \phi_{1}\left(\xi-c \tau_{4}\right)\right]=0 .
\end{array}\right.
$$


Thus, we will tackle the existence of solutions to (3.1) with the asymptotic boundary conditions

$$
\begin{cases}\lim _{\xi \rightarrow-\infty} \phi_{1}(\xi)=0, & \lim _{\xi \rightarrow+\infty} \phi_{1}(\xi)=k_{1}, \\ \lim _{\xi \rightarrow-\infty} \phi_{2}(\xi)=0, & \lim _{\xi \rightarrow+\infty} \phi_{2}(\xi)=k_{2} .\end{cases}
$$

Define $f_{c}(\phi)=\left(f_{c 1}(\phi), f_{c 2}(\phi)\right)^{T}: X_{\tau}=C\left([-c \tau, 0] ; \mathbb{R}^{2}\right) \rightarrow \mathbb{R}^{2}$ by

$$
\begin{aligned}
& f_{c 1}(\phi)=r_{1} \phi_{1}(0)\left[1-a_{1} \phi_{1}(0)-b_{1} \phi_{1}\left(-c \tau_{1}\right)+c_{1} \phi_{2}\left(-c \tau_{2}\right)\right], \\
& f_{c 2}(\phi)=r_{2} \phi_{2}(0)\left[1-a_{2} \phi_{2}(0)-b_{2} \phi_{2}\left(-c \tau_{3}\right)+c_{2} \phi_{1}\left(-c \tau_{4}\right)\right],
\end{aligned}
$$

where $\tau=\max \left\{\tau_{1}, \tau_{2}, \tau_{3}, \tau_{4}\right\}$.

Now, we prove that $f_{c}(\phi)$ satisfies $\left(\mathrm{QM}^{*}\right)$.

Lemma 3.1 For any $c>c^{*}=2 \max \left\{\sqrt{D_{1} r_{1}}, \sqrt{D_{2} r_{2}}\right\}, f_{c}(\phi)$ satisfies $\left(\mathrm{QM}^{*}\right)$ if $\tau_{1}$ and $\tau_{3}$ are sufficiently small.

Proof For any $\phi=\left(\phi_{1}, \phi_{2}\right), \psi=\left(\psi_{1}, \psi_{2}\right) \in X_{\tau}=C\left([-c \tau, 0] ; \mathbb{R}^{2}\right)$ with (i) $(0,0) \leq \psi(s) \leq$ $\phi(s) \leq\left(k_{1}, k_{2}\right)$ for $s \in[-c \tau, 0]$ and (ii) $e^{\beta_{i} s}\left[\phi_{i}(s)-\psi_{i}(s)\right]$ nondecreasing in $s \in[-c \tau, 0]$, we have

$$
\begin{aligned}
f_{c 1}(\phi) & -f_{c 1}(\psi) \\
= & r_{1} \phi_{1}(0)\left[1-a_{1} \phi_{1}(0)-b_{1} \phi_{1}\left(-c \tau_{1}\right)+c_{1} \phi_{2}\left(-c \tau_{2}\right)\right] \\
& -r_{1} \psi_{1}(0)\left[1-a_{1} \psi_{1}(0)-b_{1} \psi_{1}\left(-c \tau_{1}\right)+c_{1} \psi_{2}\left(-c \tau_{2}\right)\right] \\
= & r_{1}\left[\phi_{1}(0)-\psi_{1}(0)\right]-r_{1} a_{1}\left[\phi_{1}^{2}(0)-\psi_{1}^{2}(0)\right] \\
& -r_{1} b_{1}\left[\phi_{1}(0) \phi_{1}\left(-c \tau_{1}\right)-\psi_{1}(0) \psi_{1}\left(-c \tau_{1}\right)\right] \\
& +r_{1} c_{1}\left[\phi_{1}(0) \phi_{2}\left(-c \tau_{2}\right)-\psi_{1}(0) \psi_{2}\left(-c \tau_{2}\right)\right. \\
= & r_{1}\left[\phi_{1}(0)-\psi_{1}(0)\right]-r_{1} a_{1}\left[\phi_{1}(0)-\psi_{1}(0)\right]\left[\phi_{1}(0)+\psi_{1}(0)\right] \\
& -r_{1} b_{1}\left[\phi_{1}(0) \phi_{1}\left(-c \tau_{1}\right)-\phi_{1}(0) \psi_{1}\left(-c \tau_{1}\right)+\phi_{1}(0) \psi_{1}\left(-c \tau_{1}\right)-\psi_{1}(0) \psi_{1}\left(-c \tau_{1}\right)\right] \\
& +r_{1} c_{1}\left[\phi_{1}(0) \phi_{2}\left(-c \tau_{2}\right)-\psi_{1}(0) \phi_{2}\left(-c \tau_{2}\right)+\psi_{1}(0) \phi_{2}\left(-c \tau_{2}\right)-\psi_{1}(0) \psi_{2}\left(-c \tau_{2}\right)\right] \\
= & r_{1}\left[1-a_{1}\left(\phi_{1}(0)+\psi_{1}(0)\right)-r_{1} b_{1} \psi_{1}\left(-c \tau_{1}\right)+r_{1} c_{1} \phi_{2}\left(-c \tau_{2}\right)\right]\left[\phi_{1}(0)-\psi_{1}(0)\right] \\
& -r_{1} b_{1} \phi_{1}(0)\left[\phi_{1}\left(-c \tau_{1}\right)-\psi_{1}\left(-c \tau_{1}\right)\right]+r_{1} c_{1} \psi_{1}(0)\left[\phi_{2}\left(-c \tau_{2}\right)-\psi_{2}\left(-c \tau_{2}\right)\right] \\
\geq & r_{1}\left[1+c_{1} \phi_{2}\left(-c \tau_{2}\right)-a_{1}\left(\phi_{1}(0)+\psi_{1}(0)\right)-b_{1} \psi_{1}\left(-c \tau_{1}\right)\right. \\
& \left.-b_{1} \psi_{1}(0) e^{c \beta_{1} \tau_{1}}\right]\left[\phi_{1}(0)-\psi_{1}(0)\right] \\
\geq & r_{1}\left[1-a_{1}\left(\phi_{1}(0)+\psi_{1}(0)\right)-b_{1} k_{1}-b_{1} k_{1} e^{\beta_{1} c \tau_{1}}\right]\left[\phi_{1}(0)-\psi_{1}(0)\right] .
\end{aligned}
$$

Hence we get

$$
\begin{aligned}
& f_{c 1}(\phi)-f_{c 1}(\psi)+\beta_{1}\left[\phi_{1}(0)-\psi_{1}(0)\right] \\
& \quad \geq\left[\beta_{1}+r_{1}-a_{1} r_{1}\left(\phi_{1}(0)+\psi_{1}(0)\right)-r_{1} b_{1} k_{1}-r_{1} b_{1} k_{1} e^{c \beta_{1} \tau_{1}}\right]\left[\phi_{1}(0)-\psi_{1}(0)\right] \\
& \quad \geq\left[\beta_{1}+r_{1}-2 r_{1} a_{1} k_{1}-r_{1} b_{1} k_{1}-r_{1} b_{1} k_{1} e^{\beta_{1} c \tau_{1}}\right]\left[\phi_{1}(0)-\psi_{1}(0)\right] .
\end{aligned}
$$


Similarly, we have

$$
\begin{aligned}
& f_{c 2}(\phi)-f_{c 2}(\psi)+\beta_{2}\left[\phi_{2}(0)-\psi_{2}(0)\right] \\
& \quad \geq\left[\beta_{2}+r_{2}-2 r_{2} a_{2} k_{2}-r_{2} b_{2} k_{2}-r_{2} b_{2} k_{2} e^{\beta_{2} c \tau_{3}}\right]\left[\phi_{2}(0)-\psi_{2}(0)\right] .
\end{aligned}
$$

Choose $\beta_{1}>0$ and $\beta_{2}>0$ such that

$$
\left\{\begin{array}{l}
\beta_{1}>2 r_{1} b_{1} k_{1}+2 r_{1} a_{1} k_{1}-r_{1} \\
\beta_{2}>2 r_{2} b_{2} k_{2}+2 r_{2} a_{2} k_{2}-r_{2} .
\end{array}\right.
$$

Thus we have

$$
\left\{\begin{array}{l}
\beta_{1}+r_{1}-r_{1} b_{1} k_{1}-2 r_{1} a_{1} k_{1}-r_{1} b_{1} k_{1} e^{\beta_{1} c \tau_{1}} \geq 0 \\
\beta_{2}+r_{2}-r_{2} b_{2} k_{2}-2 r_{2} a_{2} k_{2}-r_{2} b_{2} k_{2} e^{\beta_{2} c \tau_{3}} \geq 0
\end{array}\right.
$$

if $\tau_{1}$ and $\tau_{3}$ are sufficiently small. Therefore $f_{c}(\phi)=\left(f_{c 1}(\phi), f_{c 2}(\phi)\right)$ satisfies $\left(\mathrm{QM}^{*}\right)$. This completes the proof.

Next, we construct a pair of suitable upper and lower solutions. For $c>c^{*}=$ $2 \max \left\{\sqrt{D_{1} r_{1}}, \sqrt{D_{2} r_{2}}\right\}$, define

$$
\Delta_{1}(\lambda, c)=D_{1} \lambda^{2}-c \lambda+r_{1}, \quad \Delta_{2}(\lambda, c)=D_{2} \lambda^{2}-c \lambda+r_{2} .
$$

Thus we have

$$
\Delta_{1}(\lambda, c) \begin{cases}>0 & \text { for } \lambda \in\left(0, \lambda_{1}(c)\right) \cup\left(\lambda_{2}(c),+\infty\right), \\ <0 & \text { for } \lambda \in\left(\lambda_{1}(c), \lambda_{2}(c)\right)\end{cases}
$$

and

$$
\Delta_{2}(\lambda, c) \begin{cases}>0 & \text { for } \lambda \in\left(0, \lambda_{3}(c)\right) \cup\left(\lambda_{4}(c),+\infty\right), \\ <0 & \text { for } \lambda \in\left(\lambda_{3}(c), \lambda_{4}(c)\right)\end{cases}
$$

where

$$
\begin{array}{ll}
\lambda_{1}(c):=\frac{c-\sqrt{c^{2}-4 D_{1} r_{1}}}{2 D_{1}}, & \lambda_{2}(c):=\frac{c+\sqrt{c^{2}-4 D_{1} r_{1}}}{2 D_{1}}, \\
\lambda_{3}(c):=\frac{c-\sqrt{c^{2}-4 D_{2} r_{2}}}{2 D_{2}}, & \lambda_{4}(c):=\frac{c+\sqrt{c^{2}-4 D_{2} r_{2}}}{2 D_{2}} .
\end{array}
$$

Let

$$
\eta \in\left(1, \min \left\{\frac{\lambda_{2}(c)}{\lambda_{1}(c)}, \frac{\lambda_{4}(c)}{\lambda_{3}(c)}, \frac{\lambda_{1}(c)+\lambda_{3}(c)}{\lambda_{1}(c)}, \frac{\lambda_{1}(c)+\lambda_{3}(c)}{\lambda_{3}(c)}\right\}\right)
$$

and $q>\max \left\{q_{1}, q_{2}, q_{3}\right\}$, where

$$
q_{1}=\max \left\{\left(\frac{-4 r_{1}}{\Delta_{1}\left(\eta \lambda_{1}(c), c\right)}\right)^{\frac{\eta \lambda_{1}(c)}{\lambda_{3}}},\left(\frac{-4 r_{2}}{\Delta_{2}\left(\eta \lambda_{3}(c), c\right)}\right)^{\frac{\eta \lambda_{3}(c)}{\lambda_{1}}}, 1\right\},
$$




$$
q_{2}=\max \left\{\frac{4 r_{1}}{-\Delta_{1}\left(\eta \lambda_{1}(c), c\right)}, \frac{4 r_{2}}{-\Delta_{2}\left(\eta \lambda_{3}(c), c\right)}, 1\right\}
$$

and

$$
q_{3}=\max \left\{\frac{2 r_{1}\left(a_{1}+b_{1}\right) k_{1}}{-\Delta_{1}\left(\eta \lambda_{1}(c), c\right)}, \frac{2 r_{2}\left(a_{2}+b_{2}\right) k_{2}}{-\Delta_{2}\left(\eta \lambda_{3}(c), c\right)}, 1\right\} .
$$

For these constants, we define the following continuous functions:

$$
\begin{aligned}
& \bar{\phi}_{1}(\xi)= \begin{cases}k_{1}, & \xi \geq \xi_{1}, \\
k_{1}\left(e^{\lambda_{1}(c) \xi}+q e^{\eta \lambda_{1}(c) \xi}\right), & \xi<\xi_{1},\end{cases} \\
& \bar{\phi}_{2}(\xi)= \begin{cases}k_{2}, & \xi \geq \xi_{2}, \\
k_{2}\left(e^{\lambda_{3}(c) \xi}+q e^{\eta \lambda_{3}(c) \xi}\right), & \xi<\xi_{2},\end{cases}
\end{aligned}
$$

and

$$
\begin{aligned}
& \underline{\phi}_{1}(\xi)= \begin{cases}0, & \xi \geq \xi_{3}, \\
k_{1}\left(e^{\lambda_{1}(c) \xi}-q e^{\eta \lambda_{1}(c) \xi}\right), & \xi<\xi_{3},\end{cases} \\
& \underline{\phi}_{2}(\xi)= \begin{cases}0, & \xi \geq \xi_{4}, \\
k_{2}\left(e^{\lambda_{3}(c) \xi}-q e^{\eta \lambda_{3}(c) \xi}\right), & \xi<\xi_{4} .\end{cases}
\end{aligned}
$$

If $q$ is large enough, then we easily see that $-\xi_{i}>0(i=1,2,3,4)$ are large enough.

By choosing

$$
\beta_{1}>\frac{2 \lambda_{2} e^{\lambda_{2} \xi_{1}}}{1-2 e^{\lambda_{2} \xi_{1}}} \quad \text { and } \quad \beta_{2}>\frac{2 \lambda_{4} e^{\lambda_{4} \xi_{2}}}{1-2 e^{\lambda_{4} \xi_{2}}}
$$

we easily check that $\bar{\Phi}(\xi) \in \Gamma^{*}(\underline{\Phi}, \bar{\Phi})$ and $\bar{\Phi}(\xi), \underline{\Phi}(\xi)$ satisfy (P1)-(P3). In view of (3.3) and (3.5), we choose

$$
\beta_{1}>\max \left\{2 r_{1} b_{1} k_{1}+2 r_{1} a_{1} k_{1}-r_{1}, \frac{2 \lambda_{2} e^{\lambda_{2} \xi_{1}}}{1-2 e^{\lambda_{2} \xi_{1}}}\right\}
$$

and

$$
\beta_{2}>\max \left\{2 r_{2} b_{2} k_{2}+2 r_{2} a_{2} k_{2}-r_{2}, \frac{2 \lambda_{4} e^{\lambda_{4} \xi_{2}}}{1-2 e^{\lambda_{4} \xi_{2}}}\right\} .
$$

Next, it suffices to prove that $\bar{\Phi}(\xi)$ and $\Phi(\xi)$ are a pair of upper and lower solutions of (3.1), respectively.

Lemma 3.2 If $\tau_{1}$ and $\tau_{3}$ are sufficiently small, then $\bar{\Phi}(\xi)=\left(\bar{\phi}_{1}(\xi), \bar{\phi}_{2}(\xi)\right)$ is an upper solution of (3.1).

Proof For $\bar{\phi}_{1}(\xi)$, it suffices to prove that

$$
D_{1} \bar{\phi}_{1}^{\prime \prime}(\xi)-c \bar{\phi}_{1}^{\prime}(\xi)+r_{1} \bar{\phi}_{1}(\xi)\left[1-a_{1} \bar{\phi}_{1}(\xi)-b_{1} \bar{\phi}_{1}\left(\xi-c \tau_{1}\right)+c_{1} \bar{\phi}_{2}\left(\xi-c \tau_{2}\right)\right] \leq 0
$$

for $\xi \in \mathbb{R} \backslash \xi_{1}$. 
If $\xi>\xi_{1}+c \tau_{1}$, then $\bar{\phi}_{1}(\xi)=\bar{\phi}_{1}\left(\xi-c \tau_{1}\right)=k_{1}, \bar{\phi}_{2}\left(\xi-c \tau_{2}\right) \leq k_{2}$. Thus we get

$$
\begin{aligned}
& D_{1} \bar{\phi}_{1}^{\prime \prime}(\xi)-c \bar{\phi}_{1}^{\prime}(\xi)+r_{1} \bar{\phi}_{1}(\xi)\left[1-a_{1} \bar{\phi}_{1}(\xi)-b_{1} \bar{\phi}_{1}\left(\xi-c \tau_{1}\right)+c_{1} \bar{\phi}_{2}\left(\xi-c \tau_{2}\right)\right] \\
& \quad \leq r_{1} k_{1}\left[1-\left(a_{1}+b_{1}\right) k_{1}+c_{1} \bar{\phi}_{2}\left(\xi-c \tau_{2}\right)\right] \\
& \quad \leq r_{1} k_{1}\left[1-\left(a_{1}+b_{1}\right) k_{1}+c_{1} k_{2}\right]=0 .
\end{aligned}
$$

If $\xi_{1}<\xi<\xi_{1}+c \tau_{1}$, then $\bar{\phi}_{1}(\xi)=k_{1}, \bar{\phi}_{1}\left(\xi-c \tau_{1}\right) \leq k_{1}\left(e^{\lambda_{1}(c)\left(\xi-c \tau_{1}\right)}+q e^{\eta \lambda_{1}(c)\left(\xi-c \tau_{1}\right)}\right), \bar{\phi}_{2}(\xi-$ $\left.c \tau_{2}\right) \leq k_{2}$. Thus

$$
\begin{gathered}
D_{1} \bar{\phi}_{1}^{\prime \prime}(\xi)-c \bar{\phi}_{1}^{\prime}(\xi)+r_{1} \bar{\phi}_{1}(\xi)\left[1-a_{1} \bar{\phi}_{1}(\xi)-b_{1} \bar{\phi}_{1}\left(\xi-c \tau_{1}\right)+c_{1} \bar{\phi}_{2}\left(\xi-c \tau_{2}\right)\right] \\
\leq r_{1} k_{1}\left[1-a_{1} k_{1}-b_{1} k_{1}\left(e^{\lambda_{1}(c)\left(\xi-c \tau_{1}\right)}+q e^{\eta \lambda_{1}(c)\left(\xi-c \tau_{1}\right)}\right)+c_{1} k_{2}\right] \leq 0
\end{gathered}
$$

for $\xi=\xi_{1}+c \tau_{1}$. Since $\bar{\phi}_{1}^{\prime \prime}(\xi), \bar{\phi}_{1}^{\prime}(\xi), \bar{\phi}_{1}(\xi)$, and $\bar{\phi}_{2}(\xi)$ are uniformly bounded and uniformly continuous for $\xi \in \mathbb{R} \backslash\left\{\xi_{1}, \xi_{2}\right\}$, (3.6) still holds for $\xi_{1}<\xi<\xi_{1}+c \tau_{1}$ and small enough $\tau_{1}$.

If $\xi<\xi_{1}$, then $\bar{\phi}_{1}(\xi)=k_{1}\left(e^{\lambda_{1}(c) \xi}+q e^{\eta \lambda_{1}(c) \xi}\right), \bar{\phi}_{1}\left(\xi-c \tau_{1}\right)=k_{1}\left(e^{\lambda_{1}(c)\left(\xi-c \tau_{1}\right)}+q e^{\eta \lambda_{1}(c)\left(\xi-c \tau_{1}\right)}\right)$, $\bar{\phi}_{2}\left(\xi-c \tau_{2}\right) \leq k_{2}\left(e^{\lambda_{3}(c)\left(\xi-c \tau_{2}\right)}+q e^{\eta \lambda_{3}(c)\left(\xi-c \tau_{2}\right)}\right)$, which yields

$$
\begin{aligned}
D_{1} & \bar{\phi}_{1}^{\prime \prime}(\xi)-c \bar{\phi}_{1}^{\prime}(\xi)+r_{1} \bar{\phi}_{1}(\xi)\left[1-a_{1} \bar{\phi}_{1}(\xi)-b_{1} \bar{\phi}_{1}\left(\xi-c \tau_{1}\right)+c_{1} \bar{\phi}_{2}\left(\xi-c \tau_{2}\right)\right] \\
\leq & D_{1} k_{1}\left[\lambda_{1}^{2} e^{\lambda_{1} \xi}+q\left(\eta \lambda_{1}\right)^{2} e^{\eta \lambda_{1} \xi}\right]-c k_{1}\left[\lambda_{1} e^{\lambda_{1} \xi}+q \eta \lambda_{1} e^{\eta \lambda_{1} \xi}\right] \\
& +r_{1} k_{1}\left(e^{\lambda_{1} \xi}+q e^{\eta \lambda_{1} \xi}\right)\left\{1-a_{1} k_{1}\left(e^{\lambda_{1} \xi}+q e^{\eta \lambda_{1} \xi}\right)\right. \\
& \left.-b_{1} k_{1}\left(e^{\lambda_{1}\left(\xi-c \tau_{1}\right)}+q e^{\eta \lambda_{1}\left(\xi-c \tau_{1}\right)}\right)+c_{1} k_{2}\left(e^{\lambda_{3}\left(\xi-c \tau_{2}\right)}+q e^{\eta \lambda_{3}\left(\xi-c \tau_{2}\right)}\right)\right\} \\
= & k_{1} e^{\lambda_{1} \xi}\left(D_{1} \lambda_{1}^{2}-c \lambda_{1}+r_{1}\right)+q k_{1} e^{\eta \lambda_{1} \xi}\left(D_{1}\left(\eta \lambda_{1}\right)^{2}-c \eta \lambda_{1}+r_{1}\right) \\
& +r_{1} k_{1}\left(e^{\lambda_{1} \xi}+q e^{\eta \lambda_{1} \xi}\right)\left\{-a_{1} k_{1}\left(e^{\lambda_{1} \xi}+q e^{\eta \lambda_{1} \xi}\right)-b_{1} k_{1}\left(e^{\lambda_{1}\left(\xi-c \tau_{1}\right)}+q e^{\eta \lambda_{1}\left(\xi-c \tau_{1}\right)}\right)\right. \\
& \left.+c_{1} k_{2}\left(e^{\lambda_{3}\left(\xi-c \tau_{2}\right)}+q e^{\eta \lambda_{3}\left(\xi-c \tau_{2}\right)}\right)\right\} \\
= & \Delta_{1}\left(\eta \lambda_{1}, c\right) q k_{1} e^{\eta \lambda_{1} \xi}+r_{1} k_{1}\left(e^{\lambda_{1} \xi}+q e^{\eta \lambda_{1} \xi}\right)\left\{-a_{1} k_{1}\left(e^{\lambda_{1} \xi}+q e^{\eta \lambda_{1} \xi}\right)\right. \\
& \left.-b_{1} k_{1}\left(e^{\lambda_{1}\left(\xi-c \tau_{1}\right)}+q e^{\eta \lambda_{1}\left(\xi-c \tau_{1}\right)}\right)+c_{1} k_{2}\left(e^{\lambda_{3}\left(\xi-c \tau_{2}\right)}+q e^{\eta \lambda_{3}\left(\xi-c \tau_{2}\right)}\right)\right\} .
\end{aligned}
$$

We divide this inequality into two cases.

Case 1 . Assume that $\lambda_{1}>\lambda_{3}$ and $\tau_{2}$ is large enough. We easily see that

$$
\begin{gathered}
-a_{1} k_{1}\left(e^{\lambda_{1} \xi}+q e^{\eta \lambda_{1} \xi}\right)-b_{1} k_{1}\left(e^{\lambda_{1}\left(\xi-c \tau_{1}\right)}+q e^{\eta \lambda_{1}\left(\xi-c \tau_{1}\right)}\right) \\
+c_{1} k_{2}\left(e^{\lambda_{3}\left(\xi-c \tau_{2}\right)}+q e^{\eta \lambda_{3}\left(\xi-c \tau_{2}\right)}\right) \leq 2 e^{\lambda_{3} \xi}
\end{gathered}
$$

for all $\xi<\xi_{1}$. According to the definition of $q$, we have

$$
-\Delta_{1}\left(\eta \lambda_{1}, c\right) q e^{\eta \lambda_{1} \xi} \geq 4 r_{1} e^{\lambda_{3} \xi} e^{\lambda_{1} \xi}, \quad-\Delta_{1}\left(\eta \lambda_{1}, c\right) \geq 4 r_{1} e^{\lambda_{3} \xi}
$$

which implies that

$$
\Delta_{1}\left(\eta \lambda_{1}, c\right) q k_{1} e^{\eta \lambda_{1} \xi}+2 r_{1} k_{1} e^{\lambda_{3} \xi}\left(e^{\lambda_{1} \xi+q e^{\eta \lambda_{1} \xi}}\right) \leq 0 \quad \text { for } \xi<\xi_{1} .
$$

Then (3.6) holds. 
Case 2. Assume that $\lambda_{1} \leq \lambda_{3}$ and $\tau_{2}$ is large enough. Then, for $\xi<\xi_{1}$, we have

$$
a_{1} k_{1}\left(e^{\lambda_{1} \xi}+q e^{\eta \lambda_{1} \xi}\right)+b_{1} k_{1}\left(e^{\lambda_{1}\left(\xi-c \tau_{1}\right)}+q e^{\eta \lambda_{1}\left(\xi-c \tau_{1}\right)}\right) \geq c_{1} k_{2}\left(e^{\lambda_{3}\left(\xi-c \tau_{2}\right)}+q e^{\eta \lambda_{3}\left(\xi-c \tau_{2}\right)}\right) .
$$

Thus, (3.6) still holds.

Similarly, when $\tau_{3}$ is sufficiently small and $\tau_{4}$ is large enough, it follows that

$$
D_{2} \bar{\phi}_{2}^{\prime \prime}(\xi)-c \bar{\phi}_{2}^{\prime}(\xi)+r_{2} \bar{\phi}_{2}(\xi)\left[1-a_{2} \bar{\phi}_{2}(\xi)-b_{2} \bar{\phi}_{2}\left(\xi-c \tau_{3}\right)+c_{2} \bar{\phi}_{1}\left(\xi-c \tau_{4}\right)\right] \leq 0
$$

for $\xi \in \mathbb{R}$. Therefore $\bar{\Phi}(\xi)=\left(\bar{\phi}_{1}(\xi), \bar{\phi}_{2}(\xi)\right)$ is an upper solution of (3.1). This completes the proof.

Lemma 3.3 If $\tau_{1}$ and $\tau_{3}$ are sufficiently small, then $\underline{\Phi}(\xi)=\left(\underline{\phi}_{1}(\xi), \underline{\phi}_{2}(\xi)\right)$ is a lower solution of (3.1).

Proof For $\underline{\phi}_{1}(\xi)$, we need to prove the following inequality:

$$
D_{1} \underline{\phi}_{1}^{\prime \prime}(\xi)-c \underline{\phi}_{1}^{\prime}(\xi)+r_{1} \underline{\phi}_{1}(\xi)\left[1-a_{1} \underline{\phi}_{1}(\xi)-b_{1} \underline{\phi}_{1}\left(\xi-c \tau_{1}\right)+c_{1} \underline{\phi}_{2}\left(\xi-c \tau_{2}\right)\right] \geq 0 .
$$

If $\xi>\xi_{3}$, then we easily see that $\underline{\phi}_{1}(\xi)=0$ and (3.9) holds.

If $\xi<\xi_{3}$, then we know that $\underline{\phi}_{1}(\xi)=k_{1}\left(e^{\lambda_{1} \xi}-q e^{\eta \lambda_{1} \xi}\right), \underline{\phi}_{1}\left(\xi-c \tau_{1}\right)=k_{1}\left(e^{\lambda_{1}\left(\xi-c \tau_{1}\right)}-\right.$ $\left.q e^{\eta \lambda_{1}\left(\xi-c \tau_{1}\right)}\right)$ and $\underline{\phi}_{2}\left(\xi-c \tau_{2}\right) \geq 0$. Thus

$$
\begin{aligned}
& D_{1} \underline{\phi}_{1}^{\prime \prime}(\xi)-c \underline{\phi}_{1}^{\prime}(\xi)+r_{1} \underline{\phi}_{1}(\xi)\left[1-a_{1} \underline{\phi}_{1}(\xi)-b_{1} \underline{\phi}_{1}\left(\xi-c \tau_{1}\right)+c_{1} \underline{\phi}_{2}\left(\xi-c \tau_{2}\right)\right] \\
& \geq D_{1} k_{1}\left(\lambda_{1}^{2} e^{\lambda_{1} \xi}-q\left(\eta \lambda_{1}\right)^{2} e^{\eta \lambda_{1} \xi}\right)-c k_{1}\left(\lambda_{1} e^{\lambda_{1} \xi}-q\left(\eta \lambda_{1}\right) e^{\eta \lambda_{1} \xi}\right)+r_{1} k_{1}\left(e^{\lambda_{1} \xi}-q e^{\eta \lambda_{1} \xi}\right) \\
&+r_{1} k_{1}\left(e^{\lambda_{1} \xi}-q e^{\eta \lambda_{1} \xi}\right)\left\{-a_{1} k_{1}\left(e^{\lambda_{1} \xi}-q e^{\eta \lambda_{1} \xi}\right)-b_{1} k_{1}\left(e^{\lambda_{1} \xi}-q e^{\eta \lambda_{1} \xi}\right)\right\} \\
&=-\Delta_{1}\left(\eta \lambda_{1}, c\right) q k_{1} e^{\eta \lambda_{1} \xi}-a_{1} k_{1}^{2} r_{1}\left(e^{\lambda_{1} \xi}-q e^{\eta \lambda_{1} \xi}\right)^{2} \\
&-r_{1} b_{1} k_{1}^{2}\left(e^{\lambda_{1} \xi}-q e^{\eta \lambda_{1} \xi}\right)\left(e^{\lambda_{1}\left(\xi-c \tau_{1}\right)}-q e^{\eta \lambda_{1}\left(\xi-c \tau_{1}\right)}\right) \\
& \geq-\Delta_{1}\left(\eta \lambda_{1}, c\right) q k_{1} e^{\eta \lambda_{1} \xi}-2 r_{1}\left(a_{1}+b_{1}\right) k_{1}^{2}\left(e^{\lambda_{1} \xi}-q e^{\eta \lambda_{1} \xi}\right)^{2} \\
&>-\Delta_{1}\left(\eta \lambda_{1}, c\right) q k_{1} e^{\eta \lambda_{1} \xi}-2 r_{1}\left(a_{1}+b_{1}\right) k_{1}^{2} e^{2 \lambda_{1} \xi} .
\end{aligned}
$$

By the definition of $q_{3}$ we easily see that $-\Delta_{1}\left(\eta \lambda_{1}, c\right) q k_{1} e^{\eta \lambda_{1} \xi}-2 r_{1}\left(a_{1}+b_{1}\right) k_{1}^{2} e^{2 \lambda_{1} \xi}>0$. Then we obtain

$$
D_{1} \underline{\phi}_{1}^{\prime \prime}(\xi)-c \underline{\phi}_{1}^{\prime}(\xi)+r_{1} \underline{\phi}_{1}(\xi)\left[1-a_{1} \underline{\phi}_{1}(\xi)-b_{1} \underline{\phi}_{1}\left(\xi-c \tau_{1}\right)+c_{1} \underline{\phi}_{2}\left(\xi-c \tau_{2}\right)\right] \geq 0, \quad \xi \in \mathbb{R} .
$$

Similarly, we can also prove that

$$
D_{2} \underline{\phi}_{2}^{\prime \prime}(\xi)-c \underline{\phi}_{2}^{\prime}(\xi)+r_{2} \underline{\phi}_{2}(\xi)\left[1-a_{2} \underline{\phi}_{2}(\xi)-b_{2} \underline{\phi}_{2}\left(\xi-c \tau_{3}\right)+c_{2} \underline{\phi}_{1}\left(\xi-c \tau_{4}\right)\right] \geq 0, \quad \xi \in \mathbb{R} .
$$

This completes the proof.

According to Lemmas 3.1-3.3, Theorem 2.1, and Remark 2.1, we have the following result. 
Theorem 3.1 (Existence) Assume that $\left(a_{1}+b_{1}\right)\left(a_{2}+b_{2}\right)-c_{1} c_{2}>0$. If $\tau_{1}$ and $\tau_{3}$ are suffciently small, then for every $c>2 \max \left\{\sqrt{D_{1} r_{1}}, \sqrt{D_{2} r_{2}}\right\}$, equation (1.8) has a nondecreasing traveling wave front $\left(\phi_{1}(x+c t), \phi_{2}(x+c t)\right)$ connecting $(0,0)$ to

$$
\left(k_{1}, k_{2}\right) \triangleq\left(\frac{a_{2}+b_{2}+c_{1}}{\left(a_{1}+b_{1}\right)\left(a_{2}+b_{2}\right)-c_{1} c_{2}}, \frac{a_{1}+b_{1}+c_{2}}{\left(a_{1}+b_{1}\right)\left(a_{2}+b_{2}\right)-c_{1} c_{2}}\right)
$$

and satisfying $(0,0)<\left(\phi_{1}(\xi), \phi_{2}(\xi)\right)<\left(k_{1}, k_{2}\right)$ for all $\xi \in \mathbb{R}$.

From Theorem 3.1 we obtain the following result for (1.9).

Corollary 3.1 If $\tau$ is sufficiently small, then for every $c>2 \sqrt{D r}$, equation (1.9) has a nondecreasing traveling wave front $\phi(x+c t)$ connecting 0 to $\frac{1}{a+b}$ and satisfying $0<\phi(\xi)<\frac{1}{a+b}$ for all $\xi \in \mathbb{R}$.

Remark 3.1 By choosing suitable parameters we can extend our results to Theorem 5.1.5 in [38] and Theorem 3.1 in [12].

Remark 3.2 By adopting the limiting arguments in [24, 32] Corollary 3.1 still holds for $c=2 \sqrt{D r}$. In further work, we will discuss necessary and sufficient conditions for (1.9) to have monotone traveling waves.

\section{Asymptotic behavior of traveling wave fronts}

In this section, the a priori asymptotic behavior of any nondecreasing traveling wave front is exactly derived with the help of Ikehara's theorem. We assume that $\left(a_{1}+b_{1}\right)\left(a_{2}+\right.$ $\left.b_{2}\right)>c_{1} c_{2}$ and $\left(\phi_{1}(x+c t), \phi_{2}(x+c t)\right)$ is any traveling wave front of (1.8) connecting $\mathbf{0}:=(0,0)$ to $\mathbf{K}:=\left(k_{1}, k_{2}\right)$ with wave speed $c>2 \max \left\{\sqrt{D_{1} r_{1}}, \sqrt{D_{2} r_{2}}\right\}$ and satisfying $(0,0)<$ $\left(\phi_{1}(\xi), \phi_{2}(\xi)\right)<\left(k_{1}, k_{2}\right)$ for all $\xi \in \mathbb{R}$.

By the monotonicity of traveling wave fronts the following result is clear.

Lemma 4.1 We have $\lim _{\xi \rightarrow-\infty}\left(\phi_{1}^{\prime}(\xi), \phi_{2}^{\prime}(\xi)\right)=(0,0)$ for all $\xi \in \mathbb{R}$.

Lemma 4.2 For all $\xi \in \mathbb{R}, \int_{-\infty}^{\xi} \phi_{i}(s) d s<\infty$ and $\int_{-\infty}^{\xi} \int_{-\infty}^{s} \phi_{i}(t) d t d s<\infty, i=1,2$.

Proof Since $\lim _{\xi \rightarrow-\infty}\left(\phi_{1}(\xi), \phi_{2}(\xi)\right)=(0,0)$, there exists a negative large number $\xi^{\prime}<0$ such that, for all $\xi<\xi^{\prime}$,

$$
\begin{aligned}
& D_{1} \phi_{1}^{\prime \prime}(\xi)-c \phi_{1}(\xi)+\frac{r_{1}}{2} \phi_{1}(\xi) \\
& \quad \leq D_{1} \phi_{1}^{\prime \prime}(\xi)-c \phi_{1}(\xi)+r_{1} \phi_{1}(\xi)\left[1-a_{1} \phi_{1}(\xi)-b_{1} \phi_{1}\left(\xi-c \tau_{1}\right)+c_{1} \phi_{2}\left(\xi-c \tau_{2}\right)\right] \\
& \quad=0
\end{aligned}
$$

which implies that

$$
0 \leq \frac{r_{1}}{2} \phi_{1}(\xi) \leq-D_{1} \phi_{1}^{\prime \prime}(\xi)+c \phi_{1}^{\prime}(\xi) \quad \text { for } \xi<\xi^{\prime}
$$


By Lemma 4.1 it is easy to see that $\lim _{\xi \rightarrow-\infty}\left(\phi_{1}^{\prime}(\xi), \phi_{2}^{\prime}(\xi)\right)=(0,0)$. Integrating (4.1) from $-\infty$ to $\xi\left(\xi<\xi^{\prime}\right)$, we have

$$
0 \leq \frac{r_{1}}{2} \int_{-\infty}^{\xi} \phi_{1}(t) d t \leq-D_{1} \phi_{1}^{\prime}(\xi)+c \phi_{1}(\xi) \text { for } \xi<\xi^{\prime}
$$

Thus, $\int_{-\infty}^{\xi} \phi_{1}(t) d t<\infty$ for all $\xi<\xi^{\prime}$.

Integrating (4.2) from $-\infty$ to $\xi\left(\xi<\xi^{\prime}\right)$, we obtain

$$
\begin{aligned}
0 & \leq \frac{r_{1}}{2} \int_{-\infty}^{\xi} \int_{-\infty}^{s} \phi_{1}(t) d t d s \\
& \leq-D_{1} \phi_{1}(\xi)+c \int_{-\infty}^{\xi} \phi_{1}(t) d t \\
& \leq c \int_{-\infty}^{\xi} \phi_{1}(t) d t \text { for } \xi<\xi^{\prime},
\end{aligned}
$$

which implies that $\int_{-\infty}^{\xi} \int_{-\infty}^{s} \phi_{1}(t) d t d s<\infty$ for $\xi<\xi^{\prime}$.

Thus, for any given $\xi, \int_{-\infty}^{\xi} \phi_{1}(t) d t<\infty$ and $\int_{-\infty}^{\xi} \int_{-\infty}^{s} \phi_{1}(t) d t d s<\infty$.

Similarly, we can prove that $\int_{-\infty}^{\xi} \phi_{2}(t) d t<\infty$ and $\int_{-\infty}^{\xi} \int_{-\infty}^{s} \phi_{2}(t) d t d s<\infty$ for any given $\xi$. This completes the proof.

Given a continuous function $\phi: \mathbb{R} \rightarrow \mathbb{R}$, define the two-side Laplace transform as follows:

$$
L(\lambda, \phi)=\int_{-\infty}^{+\infty} e^{-\lambda \xi} \phi(\xi) d \xi
$$

We have the following lemma.

Lemma 4.3 Assume that $\left(a_{1}+b_{1}\right)\left(a_{2}+b_{2}\right)>c_{1} c_{2}$ and $\left(\phi_{1}(\xi), \phi_{2}(\xi)\right)$ is any solution of (3.1) and (3.2) satisfying $(0,0)<\left(\phi_{1}(\xi), \phi_{2}(\xi)\right)<\left(k_{1}, k_{2}\right)$. Then:

(i) there exists $\lambda^{\prime}>0$ such that $\sup _{\xi \in \mathbb{R}} \phi_{1}(\xi) e^{-\lambda^{\prime} \xi}<\infty$ and $\left|L\left(\lambda, \phi_{1}\right)\right|<\infty$ for all $\mathfrak{R} \lambda \in\left(0, \lambda^{\prime}\right)$;

(ii) there exists $\lambda^{\prime \prime}>0$ such that $\sup _{\xi \in \mathbb{R}} \phi_{2}(\xi) e^{-\lambda^{\prime \prime} \xi}<\infty$ and $\left|L\left(\lambda, \phi_{2}\right)\right|<\infty$ for all $\Re \lambda \in\left(0, \lambda^{\prime \prime}\right)$.

Here and in what follows, $\Re \lambda$ denotes the real part of $\lambda$.

Proof We only need to prove (i). Similarly, we can prove (ii). To do this, we first show that there exists $\lambda^{\prime}>0$ such that $\sup _{\xi \in \mathbb{R}} \phi_{1}(\xi) e^{-\lambda^{\prime} \xi}<\infty$. Letting $w(\xi)=\int_{-\infty}^{\xi} \phi_{1}(t) d t$, it follows that $0<w(\xi)<\infty$ for all $\xi \in \mathbb{R}$, and $w(\xi)$ is nondecreasing on $\xi$. According to (4.3), for any $l>0$, we have

$$
0 \leq r_{1} l w(\xi-l) \leq r_{1} \int_{\xi-l}^{\xi} w(t) d t \leq r_{1} \int_{-\infty}^{\xi} w(t) d t \leq 2 c w(\xi)
$$

for $\xi<\xi^{\prime}$, where $\xi^{\prime}$ is given in Lemma 4.2. Therefore, there exists a constant $l_{0}>0$ such that $0<\frac{2 c}{l_{0} r_{1}}<1$ and $w\left(\xi-l_{0}\right)<\frac{2 c}{l_{0} r_{1}} w(\xi)$ for $\xi<\xi^{\prime}$. 
Let $m(\xi)=w(\xi) e^{-\lambda^{\prime} \xi}$, where $\lambda^{\prime}=\frac{1}{l_{0}} \ln \frac{l_{0} r_{1}}{2 c}>0$. Then

$$
\begin{aligned}
m\left(\xi-l_{0}\right) & =w\left(\xi-l_{0}\right) e^{-\lambda^{\prime}\left(\xi-l_{0}\right)}=w\left(\xi-l_{0}\right) e^{-\lambda^{\prime} \xi} e^{\lambda^{\prime} l_{0}} \\
& =\frac{l_{0} r_{1}}{2 c} w\left(\xi-l_{0}\right) e^{-\lambda^{\prime} \xi} \leq w(\xi) e^{-\lambda^{\prime} \xi}=m(\xi) \quad \text { for } \xi<\xi^{\prime}
\end{aligned}
$$

Note that $m(\xi)$ is bounded for $\xi \in\left[\xi^{\prime}-l_{0}, \xi^{\prime}\right]$. Then (4.4) implies that $m(\xi)$ is bounded for all $\xi \leq \xi^{\prime}$. Therefore, it follows from the continuity of $m(\xi)$ that $m(\xi)$ is bounded for all $\xi \leq 0$. Integrating $\phi_{1}(\xi)$ from 0 to $\xi$ for $\xi>0$, we have

$$
w(\xi)-w(0)=\int_{0}^{\xi} \phi_{1}(s) d s \leq k_{1} \xi
$$

which implies that $0 \leq w(\xi) \leq w(0)+k_{1} \xi$. Since $\lim _{\xi \rightarrow+\infty} \xi e^{-\lambda^{\prime} \xi}=0$, we have $\lim _{\xi \rightarrow+\infty} w(\xi) e^{-\lambda^{\prime} \xi}=0$. Again, since $m(\xi)$ is bounded for all $\xi \leq 0$, it follows that $0<$ $\sup _{\xi \in \mathbb{R}} w(\xi) e^{-\lambda^{\prime} \xi}<\infty$. According to (4.3), we have

$$
0 \leq D_{1} \phi_{1}(\xi) e^{-\lambda^{\prime} \xi} \leq c w(\xi) e^{-\lambda^{\prime} \xi}
$$

for all $\xi<\xi^{\prime}$. On the other hand, it is easy to see that $\lim _{\xi \rightarrow+\infty} \phi_{1}(\xi) e^{-\lambda^{\prime} \xi}=0$, since $\phi_{1}(\xi)$ is bounded. Therefore $0<\sup _{\xi \in \mathbb{R}} \phi_{1}(\xi) e^{-\lambda^{\prime} \xi}<\infty$.

Next, we prove that $\left|L\left(\lambda, \phi_{1}\right)\right|<\infty$ for all $\Re \lambda \in\left(0, \lambda^{\prime}\right)$. Indeed, letting $M=\sup _{\xi \in \mathbb{R}} \phi_{1}(\xi) \times$ $e^{-\lambda^{\prime} \xi}$, we have $0<\phi_{1}(\xi) \leq M e^{\lambda^{\prime} \xi}$ for all $\xi \in \mathbb{R}$. Since

$$
0<\left|\int_{0}^{+\infty} \phi_{1}(\xi) e^{-\lambda \xi} d \xi\right|<k_{1} \int_{0}^{+\infty} e^{-\Re \lambda \xi} d \xi=\frac{k_{1}}{\Re \lambda}
$$

and

$$
\left|\int_{-\infty}^{0} \phi_{1}(\xi) e^{-\lambda \xi} d \xi\right| \leq \int_{-\infty}^{0} M e^{\left(\lambda^{\prime}-\Re \lambda\right) \xi} d \xi=\frac{M}{\lambda^{\prime}-\Re \lambda}
$$

for all $\Re \lambda \in\left(0, \lambda^{\prime}\right)$, we get

$$
\left|L\left(\lambda, \phi_{1}\right)\right|=\left|\int_{0}^{+\infty} \phi_{1}(\xi) e^{-\lambda \xi} d \xi+\int_{-\infty}^{0} \phi_{1}(\xi) e^{-\lambda \xi} d \xi\right|<\infty
$$

Similarly, we can prove that there is a positive number $\lambda^{\prime \prime}>0$ such that $\left|L\left(\lambda, \phi_{2}\right)\right|<\infty$ for all $\Re \lambda \in\left(0, \lambda^{\prime \prime}\right)$. This completes the proof.

To study the asymptotic behavior of the solutions $\left(\phi_{1}(\xi), \phi_{2}(\xi)\right)$ at the minus infinity, we need the following version of Ikehara's theorem [2].

Proposition 4.1 (Ikehara's theorem) Let $F(\lambda)=\int_{0}^{+\infty} \phi(\xi) e^{-\lambda \xi} d \xi$ with positive decreasing function $\phi(\xi)$. Assume that $F(\lambda)$ has the representation

$$
F(\lambda)=\frac{H(\lambda)}{(\lambda+\alpha)^{k+1}}
$$


where $k>-1, \alpha>0$, and $H(\lambda)$ is analytic in the strip $-\alpha \leq \Re \lambda<0$. Then

$$
\lim _{\xi \rightarrow-\infty} \frac{\phi(\xi)}{|\xi|^{k} e^{-\alpha \xi}}=\frac{H(-\alpha)}{\Gamma(\alpha+1)}
$$

Theorem 4.1 (Asymptotic behavior) Assume that $\left(a_{1}+b_{1}\right)\left(a_{2}+b_{2}\right)>c_{1} c_{2}$. If for $c>$ $2 \max \left\{\sqrt{D_{1} r_{1}}, \sqrt{D_{2} r_{2}}\right\},\left(\phi_{1}(\xi), \phi_{2}(\xi)\right)$ is any traveling wave front of $(1.8)$ connecting $(0,0)$ to $\left(k_{1}, k_{2}\right)$ and satisfying $(0,0)<\left(\phi_{1}(\xi), \phi_{2}(\xi)\right)<\left(k_{1}, k_{2}\right)$, then there exist two constants $h_{1}$ and $h_{2}$ such that

$$
\lim _{\xi \rightarrow-\infty}\left(\phi_{1}(\xi) e^{-\lambda_{1}\left(\xi+h_{1}\right)}, \phi_{2}(\xi) e^{-\lambda_{3}\left(\xi+h_{2}\right)}\right)=(1,1)
$$

where $\lambda_{1}$ and $\lambda_{3}$ are defined in Sect. 3 , that is,

$$
\lambda_{1}=\frac{c-\sqrt{c^{2}-4 D_{1} r_{1}}}{2 D_{1}} \text { and } \lambda_{3}=\frac{c-\sqrt{c^{2}-4 D_{2} r_{2}}}{2 D_{2}} .
$$

Proof According to the wave profile equations (3.1), it follows that

$$
\begin{aligned}
& \left(D_{1} \lambda^{2}-c \lambda+r_{1}\right) L_{1}\left(\lambda, \phi_{1}\right) \\
& \quad=r_{1} \int_{-\infty}^{\infty} e^{-\lambda \xi}\left[a_{1} \phi_{1}^{2}(\xi)+b_{1} \phi_{1}(\xi) \phi_{1}\left(\xi-c \tau_{1}\right)-c_{1} \phi_{1}(\xi) \phi_{2}\left(\xi-c \tau_{2}\right)\right] d \xi \\
& \quad=: r_{1} \int_{-\infty}^{\infty} e^{-\lambda \xi} \mathcal{Q}_{1}\left(\phi_{1}, \phi_{2}\right)(\xi) d \xi
\end{aligned}
$$

and

$$
\begin{aligned}
& \left(D_{2} \lambda^{2}-c \lambda+r_{2}\right) L_{2}\left(\lambda, \phi_{2}\right) \\
& \quad=r_{2} \int_{-\infty}^{\infty} e^{-\lambda \xi}\left[a_{2} \phi_{2}^{2}(\xi)+b_{2} \phi_{2}(\xi) \phi_{2}\left(\xi-c \tau_{3}\right)-c_{2} \phi_{2}(\xi) \phi_{1}\left(\xi-c \tau_{4}\right)\right] d \xi \\
& \quad=: r_{2} \int_{-\infty}^{\infty} e^{-\lambda \xi} \mathcal{Q}_{2}\left(\phi_{1}, \phi_{2}\right)(\xi) d \xi .
\end{aligned}
$$

It is easily seen that the left-hand sides of (4.5) and (4.6) are analytic for $\Re \lambda \in\left(0, \lambda^{\prime}\right)$ and $\Re \lambda \in\left(0, \lambda^{\prime \prime}\right)$, respectively. Note that

$$
\left|\int_{-\infty}^{\infty} e^{-\lambda \xi} \phi_{1}^{2}(\xi) d \xi\right| \leq \int_{-\infty}^{\infty} e^{-\Re \lambda \xi} \phi_{1}^{2}(\xi) d \xi \leq \sup _{\xi \in \mathbb{R}} \phi_{1}(\xi) e^{-\lambda^{\prime} \xi} \int_{-\infty}^{\infty} e^{-\left(\Re \lambda-\lambda^{\prime}\right) \xi} \phi_{1}(\xi) d \xi<\infty
$$

for $\Re \lambda \in\left(0,2 \lambda^{\prime}\right)$. Similarly, we can obtain

$$
\left|\int_{-\infty}^{\infty} e^{-\lambda \xi} \phi_{1}(\xi) \phi_{1}\left(\xi-c \tau_{1}\right) d \xi\right|<\infty \quad \text { for } \Re \lambda \in\left(0,2 \lambda^{\prime}\right)
$$

and

$$
\left|\int_{-\infty}^{\infty} e^{-\lambda \xi} \phi_{1}(\xi) \phi_{2}\left(\xi-c \tau_{2}\right) d \xi\right|<\infty \quad \text { for } \Re \lambda \in\left(0, \lambda^{\prime}+\lambda^{\prime \prime}\right) .
$$


According to (4.7)-(4.9), it follows that

$$
\left|\int_{-\infty}^{\infty} e^{-\lambda \xi} \mathcal{Q}_{1}\left(\phi_{1}, \phi_{2}\right)(\xi) d \xi\right|<\infty \quad \text { for } \Re \lambda \in\left(0, \min \left\{2 \lambda^{\prime}, \lambda^{\prime}+\lambda^{\prime \prime}\right\}\right)
$$

Similarly, we have

$$
\left|\int_{-\infty}^{\infty} e^{-\lambda \xi} \mathcal{Q}_{2}\left(\phi_{1}, \phi_{2}\right)(\xi) d \xi\right|<\infty \quad \text { for } \Re \lambda \in\left(0, \min \left\{2 \lambda^{\prime \prime}, \lambda^{\prime}+\lambda^{\prime \prime}\right\}\right)
$$

We now use a property of the Laplace transform [36]. Since $\phi_{1}(\xi)>0$, there exists a real number $\kappa$ such that $L\left(\lambda, \phi_{1}\right)$ is analytic for $0<\Re \lambda<\kappa$ and $L\left(\lambda, \phi_{1}\right)$ has a singularity at $\mu=\kappa$. Hence, according to (4.5) and $D_{1} \lambda_{1}^{2}-c \lambda_{1}+r_{1}=0$, we have $\kappa=\lambda_{1}$, that is, $L\left(\lambda, \phi_{1}\right)$ is analytic for $0<\Re \lambda<\lambda_{1}$, and $L\left(\lambda, \phi_{1}\right)$ has a singularity at $\lambda=\lambda_{1}$. Similarly, we can prove that $L\left(\lambda, \phi_{2}\right)$ is analytic for $0<\Re \lambda<\lambda_{3}$ and $L\left(\lambda, \phi_{2}\right)$ has a singularity at $\lambda=\lambda_{3}$.

To apply Proposition 4.1 (Ikehara's theorem), we need to check that $\phi_{1}$ and $\phi_{2}$ satisfy all conditions of Ikehara's theorem. For $c>2 \max \left\{\sqrt{D_{1} r_{1}}, \sqrt{D_{2} r_{2}}\right\}$, we rewrite (4.5) as

$$
\begin{aligned}
& \int_{-\infty}^{0} \phi_{1}(\xi) e^{-\lambda \xi} d \xi \\
= & \frac{r_{1} \int_{-\infty}^{\infty} e^{-\lambda \xi} \mathcal{Q}_{1}\left(\phi_{1}, \phi_{2}\right)(\xi) d \xi}{D_{1} \lambda^{2}-c \lambda+r_{1}}-\int_{0}^{+\infty} \phi_{1}(\xi) e^{-\lambda \xi} d \xi \quad \text { for } \lambda \in\left(0, \lambda_{1}\right) .
\end{aligned}
$$

Note that $\int_{0}^{+\infty} \phi_{1}(\xi) e^{-\lambda \xi} d \xi$ is analytic for $\Re \lambda>0$ and $D_{1} \lambda^{2}-c \lambda+r_{1}=0$ does not have any positive roots with $\Re \lambda=\lambda_{1}$ other than $\lambda=\lambda_{1}$.

Let $u_{i}(\xi)=\phi_{i}(-\xi), i=1,2$, and

$$
\begin{aligned}
\mathcal{Q}_{1}\left(\phi_{1}, \phi_{2}\right)(\xi) & =a_{1} u_{1}^{2}(-\xi)+b_{1} u_{1}(-\xi) u_{1}\left(-\xi+c \tau_{1}\right)-c_{1} u_{1}(-\xi) u_{2}\left(-\xi+c \tau_{2}\right) \\
& :=\mathbf{T}\left(u_{1}, u_{2}\right)(-\xi) .
\end{aligned}
$$

It is clear that $u(\xi)$ is decreasing in $\xi$,

$$
\begin{aligned}
\int_{0}^{+\infty} e^{\lambda \theta} u_{1}(\theta) d \theta & =\frac{\int_{-\infty}^{+\infty} e^{\lambda \xi} \mathbf{T}\left(u_{1}, u_{2}\right)(\theta) d \theta}{D_{1} \lambda^{2}-c \lambda+r_{1}}-\int_{0}^{+\infty} e^{-\lambda \xi} \phi_{1}(\xi) d \xi \\
& =: \frac{H(\lambda)}{\lambda-\lambda_{1}} \neq 0,
\end{aligned}
$$

and

$$
H(\lambda)=\frac{\left(\lambda-\lambda_{1}\right) \int_{-\infty}^{\infty} e^{\lambda \theta} \mathbf{T}\left(u_{1}, u_{2}\right)(\theta) d \theta}{D_{1} \lambda^{2}-c \lambda+r_{1}}-\left(\lambda-\lambda_{1}\right) \int_{0}^{+\infty} e^{-\lambda \xi} \phi_{1}(\xi) d \xi
$$

We easily check that $\lim _{\lambda \rightarrow \lambda_{1}} H(\lambda)$ exists. Therefore $H(\lambda)$ is analytic for all $0<\Re \lambda \leq \lambda_{1}$. According to Proposition 4.1, we obtain

$$
\lim _{\xi \rightarrow+\infty} \frac{u_{1}(\xi)}{e^{-\lambda_{1} \xi}} \quad \text { exists, i.e., } \lim _{\xi \rightarrow-\infty} \frac{\phi_{1}(\xi)}{e^{\lambda_{1} \xi}} \quad \text { exists. }
$$


Similarly, we can also verify that

$$
\lim _{\xi \rightarrow-\infty} \frac{\phi_{2}(\xi)}{e^{\lambda_{3} \xi}} \quad \text { exists. }
$$

Therefore there exist two constants $h_{1}$ and $h_{2}$ such that

$$
\lim _{\xi \rightarrow-\infty}\left(\phi_{1}(\xi) e^{-\lambda_{1}\left(\xi+h_{1}\right)}, \phi_{2}(\xi) e^{-\lambda_{3}\left(\xi+h_{2}\right)}\right)=(1,1) .
$$

This completes the proof.

According to Theorem 4.1, we can obtain the following results for (1.7) and (1.9), respectively. For (1.7), taking $a_{1}=a_{2}=0$ in (1.8), we have the following:

Corollary 4.1 (Asymptotic behavior) Assume that $b_{1} b_{2}>c_{1} c_{2}$. If for $c>2 \max \left\{\sqrt{D_{1} r_{1}}\right.$, $\left.\sqrt{D_{2} r_{2}}\right\},\left(\phi_{1}(x+c t), \phi_{2}(x+c t)\right)$ is any traveling wave front of $(1.7)$ connecting $(0,0)$ to $\left(k_{1}, k_{2}\right)$ and satisfying $(0,0)<\left(\phi_{1}(\xi), \phi_{2}(\xi)\right)<\left(k_{1}, k_{2}\right)$, then there exist two constants $h_{1}$ and $h_{2}$ such that

$$
\lim _{\xi \rightarrow-\infty}\left(\phi_{1}(\xi) e^{-\lambda_{1}\left(\xi+h_{1}\right)}, \phi_{2}(\xi) e^{-\lambda_{3}\left(\xi+h_{2}\right)}\right)=(1,1)
$$

where

$$
\lambda_{1}=\frac{c-\sqrt{c^{2}-4 D_{1} r_{1}}}{2 D_{1}} \text { and } \lambda_{3}=\frac{c-\sqrt{c^{2}-4 D_{2} r_{2}}}{2 D_{2}} .
$$

For (1.9), we have the following:

Corollary 4.2 (Asymptotic behavior) If for $c>2 \sqrt{D r}, \phi(\xi)$ is any traveling wave front of (1.9) connecting 0 to $\frac{1}{a+b}$ and satisfying $0<\phi(\xi)<\frac{1}{a+b}$, then there exists a constant $h$ such that

$$
\lim _{\xi \rightarrow-\infty} \phi(\xi) e^{-\mu(\xi+h)}=1
$$

where

$$
\mu=\frac{c-\sqrt{c^{2}-4 D r}}{2 D}
$$

Remark 4.1 Letting $a=0$ and $b=1$ in (1.9), Corollary 4.2 reduces to Theorem 2.6 in [28].

Acknowledgements

The research of W.G. Zhang was partially supported by National Natural Science Foundation of China (No. 11471215), by Shanghai Leading Academic Discipline Project (No. XTKX2012), and by the Hujiang Foundation of China (B14005). The research of Z.X. Yu was partially supported by Natural Science Foundation of Shanghai (No. 18ZR1426500). We are grateful to anonymous referees and the editor for their carefully reading and helpful suggestions, which led to an improvement of our original manuscript. 
Authors' contributions

All authors have equal contributions. All authors read and approved the final manuscript.

\section{Author details}

'Business School, University of Shanghai for Science and Technology, Shanghai, P.R. China. ${ }^{2}$ College of Science, University of Shanghai for Science and Technology, Shanghai, China.

\section{Publisher's Note}

Springer Nature remains neutral with regard to jurisdictional claims in published maps and institutional affiliations.

Received: 3 January 2018 Accepted: 10 May 2018 Published online: 07 June 2018

\section{References}

1. Cantrell, R.S., Cosner, C.: Spatial Ecology Via Reaction-Diffusion Equations. Wiley, Chichester (2003)

2. Carr, J., Chmaj, A.: Uniqueness of travelling waves for nonlocal monostable equations. Proc. Am. Math. Soc. 132, 2433-2439 (2004)

3. Cushing, J.M.: Integrodifferential Equations and Delay Models in Population Dynamics. Lecture Notes in Biomath. vol. 20. Springer, Berlin (1977)

4. Fang, J., Wu, J.H.: Monotone traveling waves for delayed Lotka-Volterra competition systems. Discrete Contin. Dyn. Syst. 32, 3043-3058 (2012)

5. Fisher, R.A.: The wave of advance of advantageous gene. Ann. Eugen. 7, 355-369 (1937)

6. Gomez, A., Trofimchuk, S.: Monotone traveling wavefronts of the KPP-Fisher delayed equation. J. Differ. Equ. 250, 1767-1787 (2011)

7. Gopalsamy, K.: Stability and Oscillations in Delay Differential Equations of Population Dynamics. Kluwer Academic, London (1992)

8. Guo, J., Liang, X.: The minimal speed of traveling fronts for the Lotka-Volterra competition system. J. Dyn. Differ. Equ. 23, 353-363 (2011)

9. Hosono, Y.: The minimal speed of traveling fronts for a diffusive Lotka-Volterra competition model. Bull. Math. Biol. 60, 435-448 (1998)

10. Hou, X., Leung, A.: Traveling wave solutions for a competitive reaction-diffusion system and their asymptotics. Nonlinear Anal., Real World Appl. 9, 2196-2213 (2008)

11. Hsu, C.H., Yang, T.S.: Existence, uniqueness, monotonicity and asymptotic behavior of traveling waves for a epidemic model. Nonlinearity 26, 121-139 (2013) Corrigendum: 26, 2925-2928 (2013)

12. Huang, J., Zou, X.: Traveling wave fronts in diffusive and cooperative Lotka-Volterra system with delays. J. Math. Anal. Appl. 271, 455-466 (2002)

13. Huang, J., Zou, X.: Existence of travelling wavefronts of delayed reaction-diffusion systems without monotonicity. Discrete Contin. Dyn. Syst. 9, 925-936 (2003)

14. Huang, W.: Problem on minimum wave speed for a Lotka-Volterra reaction-diffusion competition model. J. Dyn. Differ. Equ. 22, 285-297 (2010)

15. Huang, W., Han, M.: Non-linear determinacy of minimum wave speed for a Lotka-Volterra competition model. J. Differ. Equ. 251, 1549-1561 (2011)

16. Kanel, J., Zhou, L.: Existence of wave front solutions and estimates of wave speed for a competition-diffusion system. Nonlinear Anal. TMA 27, 579-587 (1996)

17. Kolmogoroff, A., Petrovsky, I., Piscounoff, N.: Étude de l'équation de la diffusion avec croissance de la quantité de matière et son application à un problème biologique. Bull. Univ. Moscou Ser. Internationale Sec. A 1, 1-25 (1937)

18. Kuang, Y.: Delay Differential Equations with Applications in Population Dynamics. Academic Press, Boston (1993)

19. Kwong, M.K., Ou, C.: Existence and nonexistence of monotone traveling waves for the delayed Fisher equation. J. Differ. Equ. 249, 728-745 (2010)

20. Larry Rockwood, L.: Introduction to Population Ecology. Wiley, New York (2015)

21. Li, K., Li, X.: Traveling wave solutions in a delayed diffusive competition system. Nonlinear Anal. TMA 75, 3705-3722 (2012)

22. Li, W.T., Lin, G., Ruan, S.G.: Existence of traveling wave solutions in delayed reaction-diffusion systems with applications to diffusion-competition systems. Nonlinearity 19, 1253-1273 (2006)

23. Li, X.S., Lin, G.: Traveling wavefronts in nonlocal dispersal and cooperative Lotka-Volterra system with delays. Appl. Math. Comput. 204, 738-744 (2008)

24. Liang, X., Zhao, X.Q.: Asymptotic speeds of spread and traveling waves for monotone semiflows with applications. Commun. Pure Appl. Math. 60, 1-40 (2007)

25. LV, G., Wang, M.: Traveling wave front in diffusive and competitive Lotka-Volterra system with delays. Nonlinear Anal., Real World Appl. 11, 1323-1329 (2010)

26. Murray, J.D.: Mathematical Biology. Springer, New York (1993)

27. Pan, S., Li, W., Lin, G.: Travelling wave fronts in nonlocal delayed reaction-diffusion systems and applications. Z. Angew. Math. Phys. 60, 377-392 (2009)

28. Pan, S.X.: Asymptotic behavior of travelling fronts of the delayed Fisher equation. Nonlinear Anal., Real World Appl. 10, 1173-1182 (2009)

29. Seifert, G.: On a delay-differential equation for single specie population variations. Nonlinear Anal. TMA 11, 1051-1059 (1987)

30. Smith, F.E.: Population dynamics in Daphnia magna. Ecology 44, 651-663 (1963)

31. Tang, M.M., File, P.C.: Propagation fronts in competing species equations with diffusion. Arch. Ration. Mech. Anal. 73 69-77 (1978)

32. Thieme, H.R., Zhao, X.Q.: Asymptotic speeds of spread and traveling waves for integral equations and delayed reaction diffusion models. J. Differ. Equ. 195, 430-470 (2003) 
33. van-Vuuren, J.H.: The existence of traveling plane waves in a general class of competition-diffusion systems. IMA J. Appl. Math. 55, 135-148 (1995)

34. Volpert, A.I., Volpert, V.A., Volpert, V.A.: Travelling Wave Solutions of Parabolic Systems. Translations of Mathematical Monographs, vol. 140. American Mathematical Society, Providence (1994)

35. Wang, Z.C., Li, W.T., Ruan, S.: Travelling wave fronts in monostable equations with nonlocal delayed effects. J. Dyn. Differ. Equ. 20, 563-607 (2008)

36. Widder, D.V.: The Laplace Transform. Princeton University Press, Princeton (1941)

37. Wu, J.: Theorem and Applications of Partial Functional Equations. Springer, New York (1996)

38. Wu, J., Zou, X.: Traveling wave fronts of reaction-diffusion systems with delay. J. Dyn. Differ. Equ. 13, 651-687 (2001) Erratum: 61, 531-533 (2008)

39. Yu, Z.X., Mei, M.: Uniqueness and stability of traveling waves for cellular neural networks with multiple delays. J. Differ Equ. 260, 241-267 (2016)

40. Yu, Z.X., Yuan, R.: Traveling waves for a Lotka-Volterra competition system with diffusion. Math. Comput. Model. 53, 1035-1043 (2011)

41. Yu, Z.X., Yuan, R.: Existence, asymptotics and uniqueness of traveling waves for nonlocal diffusion systems with delayed nonlocal response. Taiwan. J. Math. 17, 2163-2190 (2013)

42. Zhao, X.Q.: Dynamical Systems in Population Biology. Springer, New York (2003)

\section{Submit your manuscript to a SpringerOpen ${ }^{\circ}$} journal and benefit from:

- Convenient online submission

- Rigorous peer review

- Open access: articles freely available online

- High visibility within the field

- Retaining the copyright to your article

Submit your next manuscript at $\boldsymbol{\Delta}$ springeropen.com 